\title{
Evaluation of Multi-Lead ECG Markers to Track Changes in Dispersion of Ventricular Repolarization in the Intact Human Heart
}

\author{
Michele Orini $^{1,2}$, Neil Srinivasan ${ }^{2}$, Peter Taggart $^{1}$, Pier D Lambiase $^{1,2}$ \\ ${ }^{1}$ University College London, London, United Kingdom \\ ${ }^{2}$ Barts Heart Centre, St Bartholomew's Hospital, London, United Kingdom
}

\begin{abstract}
Dispersion of ventricular repolarization (DRT) is an important factor contributing to the vulnerability to life-threatening arrhythmias. An accurate non-invasive methodology for its estimation would contribute to improve risk-prediction. We assessed 3 multi-lead ECG markers to track changes in DRT using intra-cardiac data recorded in patients with structurally normal ventricles. Changes in DRT were measured with intra-cardiac unipolar electrograms (UEG) simultaneously recorded in the $R V$ endocardium (RVendo), $L V$ endocardium (LVendo) and $L V$ epicardium (coronary sinus, LVepi) in 10 patients. Standard SIS2 restitution protocols were conducted by pacing from the RVendo $(n=8)$, LVendo $(n=10)$ and LVepi $(n=7)$. DRT was measured as latest minus earliest repolarization time (RT). In the surface ECG, DRT was estimated from precordial and augmented limb leads as: (1) Interval between the earliest and the latest maximum upslope of the T-wave $\left(\Delta T_{u p}\right)$; (2) Interval between median T-peak and median T-end ( $\left.T_{p e, m e d}\right)$; (3) Interval between the earliest T-peak and latest T-end ( $\left.T_{\text {pe,range }}\right)$. Intrapatient correlation with DRT changes was higher using $\Delta T_{u p}(0.79,0.66-0.89)$ than $T_{p e, \text { med }}(0.61,0.14-0.76$, $P=0.001)$ or $T_{p e, \text { med }}(0.71,0.44-0.79, P=0.054)$.
\end{abstract}

\section{Introduction}

Ventricular repolarization dynamics modulate vulnerability to potentially life-threatening ventricular arrhythmias. The spatial organization of ventricular repolarization and its beat to beat variability is known to play an important role in determining susceptibility to arrhythmia and its non-invasive assessment can be useful for improving risk prediction. In particular, repolarization dispersion, i.e. the duration of the repolarization process from its beginning to its end, is known to be important [1]. Unfortunately, the link between the repolarization sequence and the morphology of the T-wave of the surface ECG in the intact human heart is still poorly characterized. In this study we assessed
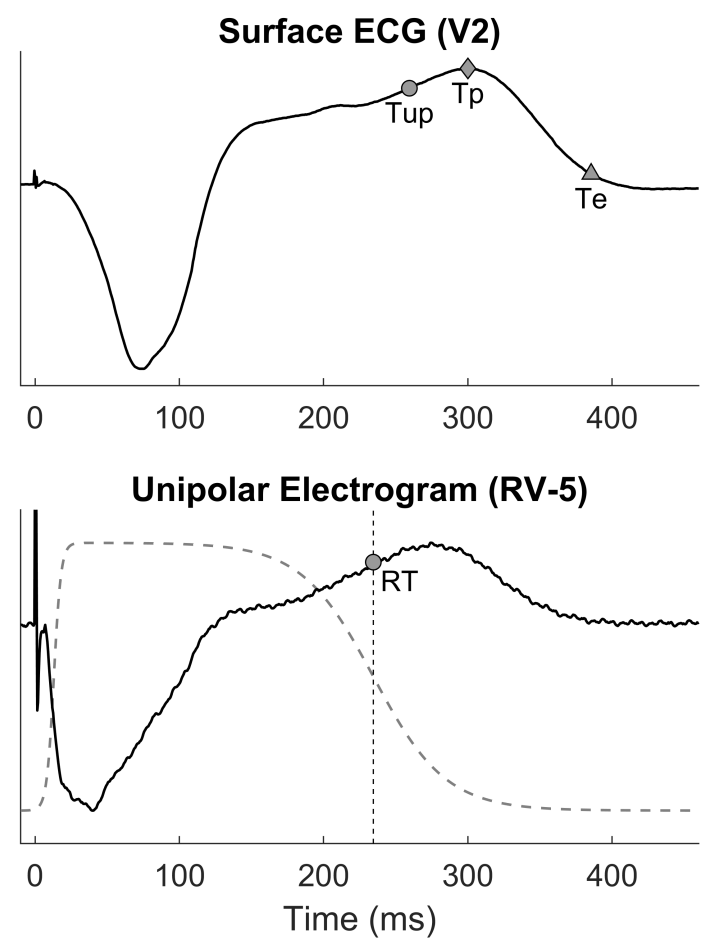

Figure 1. Repolarization markers in the surface ECG (above) and unipolar electrograms (below). Local repolarization time (RT) was measured at the maximum of the first derivative of the signal. Dashed line represents a stylized action potential.

3 multi-lead ECG markers to track changes in dispersion of repolarization using intra-cardiac data recorded in patients with structurally normal ventricles.

\section{Methods}

Three decapolar catheters were used to simultaneously map the right ventricular endocardium (RVendo), left ventricular endocardium (LVendo) and left ventricular epicardium (coronary sinus, LVepi) in 10 patients undergo- 
ing an electrophysiological study for treatment of supraventricular tachycardia [2]. Unipolar electrograms (UEG, sampling frequency $2000 \mathrm{~Hz}, 0.05-500 \mathrm{~Hz}$ ) and the 12lead ECG (sampling frequency $2000 \mathrm{~Hz}, 0.05-100 \mathrm{~Hz}$ ) were simultaneously recorded. All patients had a structurally normal heart and normal ECG. An standard S1S2 restitution protocol $[2,3]$ was conducted by pacing from the RVendo (in $n=8$ patients), LVendo (in $n=10$ patients) and LVepi (in $n=7$ patients). Following a train of nine steady-state $S 1 S 1$ stimuli at $600 \mathrm{~ms}$, an extra stimulus at a shorter coupled pacing interval $S 1 S 2$ was introduced. The $S 1 S 2$ coupling interval was decremented in 50 ms steps from $1000 \mathrm{~ms}$ to $400 \mathrm{~ms}$, then by $20 \mathrm{~ms}$ intervals between 380 and $400 \mathrm{~ms}$, and thereafter in $5 \mathrm{~ms}$ steps until the effective refractory period (ERP) of the tissue. At ERP an S2 stimulus at $10 \mathrm{~ms}+$ ERP was applied followed by further decrementing S2 in steps of $2 \mathrm{~ms}$.

Local repolarization times (RT) was measured from the UEG at the maximum of the first derivative, $\max (d V / d t)$, of the signal during the T-wave (Fig. 1). This is a standard measurement supported by experimental [4] and theoretical [5] studies, and largely adopted in human electrophysiological research $[2,6,7]$. Total dispersion of repolarization $(\Delta R T)$ was measured in the beats following the shortcoupled intervals as the interval between the earliest to the latest repolarization time (RT). Body surface ECG was analyzed with custom software as in previous studies [8,9] to measure the following ECG markers: $T_{u p}$, time of the maximum of the first derivative during the T-wave upslope, $\arg \max (d V / d t)$ (as for local RT estimate in the UEG), Twave peak (Tp) and T-wave end (Te), which was measured using the tangent method (Fig. 1). All markers were visually inspected and corrected if needed. The following markers of dispersion of repolarization were derived from inter-lead analysis of precordial and augmented limb leads of the surface ECG:

- 1. Interval between the earliest and the latest maximum upslope of the T-wave $\left(\Delta T_{u p}\right)$;

- 2. Interval between the earliest T-peak and latest T-end ( $\left.T_{\text {pe,range }}\right)$.

- 3. Interval between median T-peak and median T-end (T $\left.T_{\text {pe,med }}\right)$;

The Spearman's correlation coefficient between $\Delta R T$ and the surface ECG estimates of $\Delta R T$ was measured per each $S 1 S 2$ restitution curve $(n=25)$. Results are show as median and Q1-Q3 interval.

\section{Results}

An example of surface ECG and intra-cardiac UEG during RV pacing is shown in Fig 2. Local RT was early in the $\mathrm{RV}$-apex and late in the LV-base. As expected [5], UEGs showed upright T-wave in the RV (early RT) and inverted T-wave in the LV (late RT). Interestingly, Tup was early
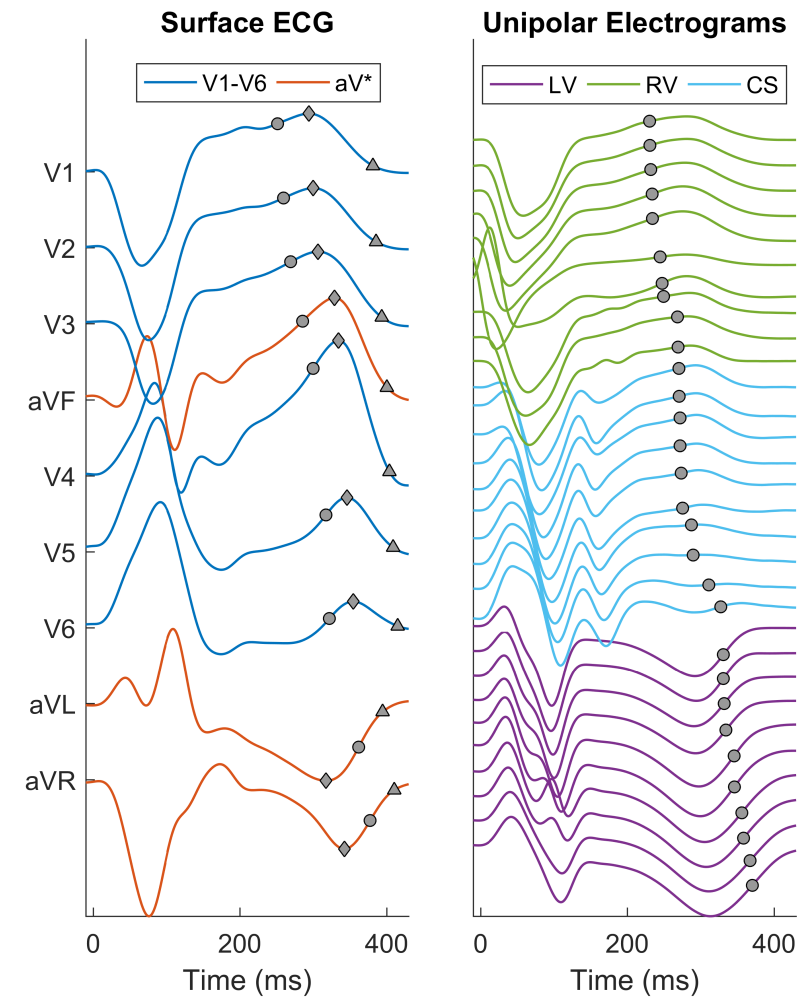

Figure 2. Intrac-ardiac (right) and corresponding surface (left) recordings during RV apical pacing. Local repolarization time (RT) is early in the RV and late in the LV (right). A similar pattern is observed for Tup on the ECG (left). Note the similar morphology between RV UEG and V1-V2.

in V1-V2, where the T-wave resembled the T-wave of RV UEGs, and late in V6 and aVL.

Representative examples of the correlation between $\Delta R T$ measured from the intrac-ardiac UEGs and estimated from the surface ECG as $\Delta T_{u p}$ is shown in Fig. 3, which shows the changes of $\Delta R T$ and $\Delta T_{u p}$ as a function of pacing interval (left) as well as their scatter-plot (right). As expected [3], dispersion of repolarization initially decreases with decreasing pacing interval and then increases for pacing interval lower than $350 \mathrm{~ms}$ as a result of the engagement of conduction velocity restitution. In both patients, the correlation coefficient was $\rho \approx 0.90$.

Group analysis showed that changes in $\Delta R T$ from cycle length equal to $1000 \mathrm{~ms}$ to effective refractory period (240, 223-250 ms) were equal to $94.4 \pm 36.9 \mathrm{~ms}$. Intrapatient correlation between changes in $\Delta R T$ and changes in surface ECG markers was equal to $0.79(0.66-0.89)$ for $\Delta T_{u p}, 0.71(0.44-0.79)$ for $T_{\text {pe,range }}$ and 0.61 $(0.14-0.76)$ for $T_{p e, m e d}$ (Fig. 4). Group differences were significant $(P=0.017$, Kruskal-Wallis test) and pair-wise comparison showed that $\Delta T_{u p}$ correlated significantly bet- 

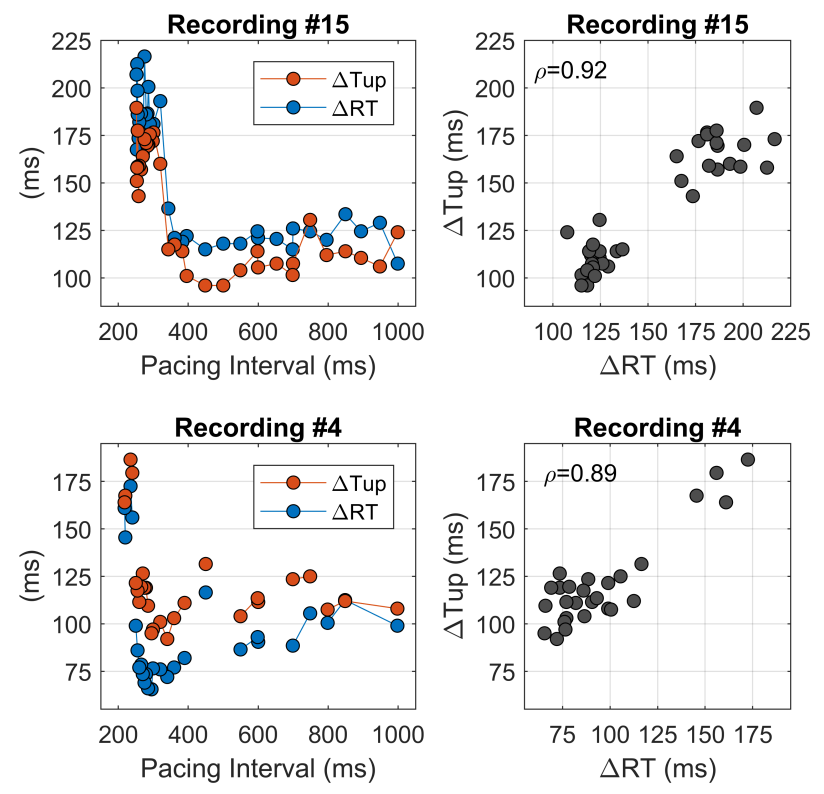

Figure 3. Relation between dispersion or repolarization measured with intra-cardiac unipolar electrograms $(\Delta R T)$ and estimated from the surface ECG as $\Delta T_{u p}$. Left: Changes of both $\Delta R T$ and $\Delta T_{u p}$ as a function of pacing interval. Right: Scatter-plot showing tight correlation between $\Delta R T$ and $\Delta T_{u p}$, with correlation coefficient $\rho>0.89$.

ter than $T_{p e, \text { med }}(P=0.001)$ and almost significantly better than $T_{\text {pe,range }}(P=0.054)$ with $\Delta R T$ (Fig. 4).

\section{Discussion}

This study assessed three surface ECG markers to track changes of dispersion of ventricular repolarization in the intact human heart. The main result is that $\Delta T_{u p}$, i.e. the interval between the earliest and latest Tup, measured as $\arg \max (d V / d t)_{\max }$, tracks changes in dispersion of repolarization with high intra-patient correlation.

The underlying hypothesis of this study is that a unipolar surface ECG lead can be interpreted as a unipolar electrogram at a distance, therefore providing information about regional repolarization of the most proximal cardiac segment. For instance, early and late RV repolarization often corresponded to upright and inverted (or biphasic) T-waves in V1-V2, respectively, while early and late LV repolarization often corresponded to upright and inverted (or biphasic) T-waves in V5-V6.

Our results are in partial agreement with the results of a recent and elegant porcine study [10].

Our study focused on markers to track changes in the dispersion of repolarization and the results were based on intra-patient correlation. This was intended to reduce the

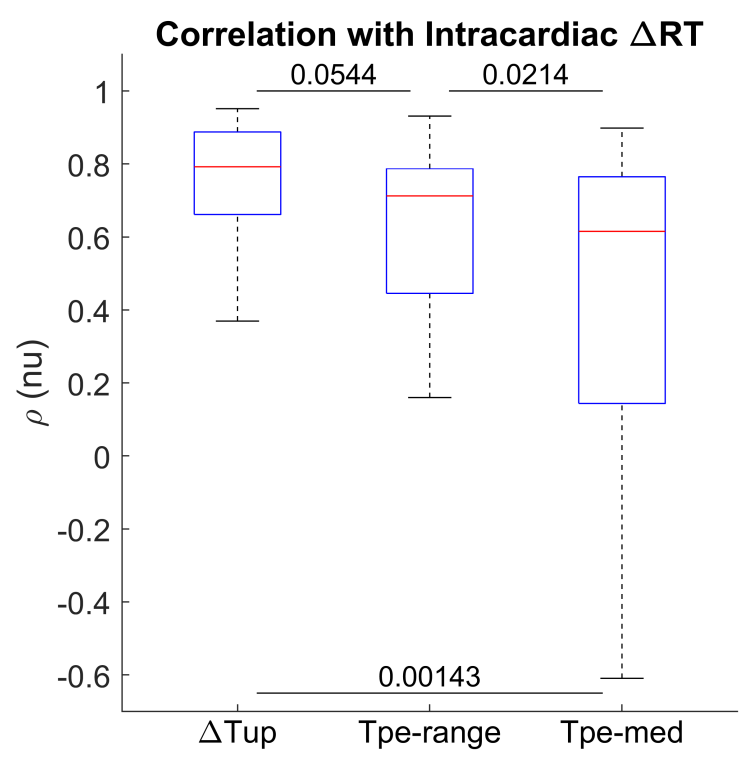

Figure 4. Distribution of the intra-patient correlation coefficient $\rho$ between dispersion or repolarization measured with intra-cardiac unipolar electrograms $(\Delta R T)$ and estimated from the surface ECG in $n=25$ restitution protocols (across 10 patients). Pair-wise different are measured with the Wilcoxon signed-rank test, with p-values reported above horizontal lines.

bias introduced by the fact that the intra-cardiac mapping did not cover the entire endocardial and epicardial surfaces of the heart, which may have resulted in an underestimation of the true dispersion of repolarization. The extent of this bias depends on the geometrical configuration of the catheters with respect to the repolarization sequence and may be different in each patient.

The results of this study have implications for cardiac risk assessment, and $\Delta T_{u p}$ should be compared and/or integrated with/to other ECG repolarization indices [11-13]. Future studies could also assess the capability of $\Delta T_{u p}$ to track beat-to-beat repolarization variability [14-16].

In conclusion, multi-lead index $\Delta T_{u p}$ tracks changes in dispersion of repolarization with high intra-patient correlation.

\section{References}

[1] Opthof T, Janse MJ, Meijborg VM, Cinca J, Rosen MR, Coronel R. Dispersion in ventricular repolarization in the human, canine and porcine heart. Progress in Biophysics and Molecular Biology jan 2016;120(1-3):222-235. ISSN 00796107.

[2] Srinivasan NT, Orini M, Simon RB, Providência R, Khan F, Segal OR, Babu G, Bradley R, Rowland E, Ahsan S, Chow AW, Lowe M, Taggart P, Lambiase P. Ventricular stimulus 
site influences dynamic dispersion of repolarization in the intact human heart. American Journal of Physiology Heart and Circulatory Physiology 2016;ajpheart.00159.2016.

[3] Orini M, Taggart P, Srinivasan N, Hayward M, Lambiase PD. Interactions between activation and repolarization restitution properties in the intact human heart: In-vivo whole-heart data and mathematical description. PLoS ONE 2016;11(9):e0161765.

[4] Coronel R, de Bakker JMT, Wilms-Schopman FJG, Opthof T, Linnenbank AC, Belterman CN, Janse MJ. Monophasic action potentials and activation recovery intervals as measures of ventricular action potential duration: Experimental evidence to resolve some controversies. Heart Rhythm 2006;3(9).

[5] Orini M, Taggart P, Lambiase PD. In vivo human sockmapping validation of a simple model that explains unipolar electrogram morphology in relation to conductionrepolarization dynamics. Journal of Cardiovascular Electrophysiology jul 2018;29(7):990-997. ISSN 15408167.

[6] Taggart P, Orini M, Hanson B, Hayward M, Clayton R, Dobrzynski H, Yanni J, Boyett M, Lambiase P. Developing a novel comprehensive framework for the investigation of cellular and whole heart electrophysiology in the in situ human heart: Historical perspectives, current progress and future prospects. Progress in Biophysics and Molecular Biology 2014;115(2-3). ISSN 00796107.

[7] Martin CA, Orini M, Srinivasan NT, Bhar-Amato J, Honarbakhsh S, Chow AW, Lowe MD, Ben-Simon R, Elliott PM, Taggart P, Lambiase PD. Assessment of a conductionrepolarisation metric to predict Arrhythmogenesis in right ventricular disorders. International Journal of Cardiology 2018;ISSN 18741754.

[8] Orini M, Blasi C, Finlay M, Hanson B, Lambiase P, Sassi R, Mainardi LT. Validation of the V-index as a metric of ventricular repolarization dispersion using intracardiac recordings. Computing in Cardiology 2015;42:673-676. ISSN 2325887X.

[9] Orini M, Tinker A, Munroe PB, Lambiase PD. Long-term intra-individual reproducibility of heart rate dynamics during exercise and recovery in the UK Biobank cohort. PLoS ONE 2017;12(9):e0183732. ISSN 19326203.

[10] Meijborg VMF, Conrath CE, Opthof T, Belterman CNW, De Bakker JMT, Coronel R. Electrocardiographic T wave and its relation with ventricular repolarization along major anatomical axes. Circulation Arrhythmia and Electrophysiology 2014;7(3):524-531. ISSN 19413084.

[11] Ramirez J, Orini M, Tucker JD, Pueyo E, Laguna P. Variability of Ventricular Repolarization Dispersion Quantified by Time-Warping the Morphology of the T-Waves. IEEE Transactions on Biomedical Engineering jul 2017; 64(7):1619-1630. ISSN 15582531.

[12] Ramírez J, Orini M, Mincholé A, Monasterio V, Cygankiewicz I, de Luna AB, Martínez JP, Pueyo E, Laguna P. T-wave morphology restitution predicts sudden cardiac death in patients with chronic heart failure. Journal of the American Heart Association 2017;6(5). ISSN 20479980.

[13] Ramírez J, Orini M, Mincholé A, Monasterio V, Cygankiewicz I, Bayés de Luna A, Martínez JP, Laguna P, Pueyo E. Sudden cardiac death and pump failure death prediction in chronic heart failure by combining ECG and clinical markers in an integrated risk model. PLoS ONE 2017; 12(10). ISSN 19326203.

[14] Hanson B, Child N, Van Duijvenboden S, Orini M, Chen Z, Coronel R, Rinaldi A, Gill J, Taggart P. Oscillatory behaviour of ventricular action potential duration in heart failure patients at respiratory rate and low frequency. Frontiers in Physiology 2014;5(OCT). ISSN 1664042X.

[15] van Duijvenboden S, Hanson B, Child N, Orini M, Rinaldi CA, Gill J, Taggart P. Effect of autonomic blocking agents on the respiratory related oscillations of ventricular action potential duration in humans. American Journal of Physiology Heart and Circulatory Physiology 2015;309(12):ajpheart.00560.2015. ISSN 1522-1539.

[16] Orini M, Pueyo E, Laguna P, Bailon R. A time-varying nonparametric methodology for assessing changes in QT variability unrelated to heart rate variability. IEEE Transactions on Biomedical Engineering 2018;65(7):1443-1451. ISSN 15582531 .

Address for correspondence:

Name: Michele Orini

Full postal address: University College London, Gower Street, London, UK.

E-mail address: m.orini@ucl.ac.uk 